This review article was published as S. Vullo, S. Kellenberger, A molecular view of the function and pharmacology of acid-sensing ion channels, Pharmacol Res (2019), doi: 10.1016/j.phrs.2019.02.005

\title{
A molecular view of the function and pharmacology of acid-sensing ion channels
}

Sabrina Vullo and Stephan Kellenberger*

Department of Pharmacology and Toxicology, University of Lausanne, Lausanne, Switzerland

* To whom correspondence should be addressed: Stephan Kellenberger, Department of Pharmacology and Toxicology, University of Lausanne, Rue du Bugnon 27, CH-1011 Lausanne, Switzerland; Stephan.Kellenberger@unil.ch; Phone +4121 692 5422, Fax $+41216925355$.

\begin{abstract}
Abbreviations
ASIC, acid-sensing ion channel; EAE, experimental autoimmune encephalomyelitis; ENaC, epithelial $\mathrm{Na}^{+}$channel; GMQ, 2-guanidine-4-methylquinazoline; LTP, long-term potentiation; MCAO, middle cerebral artery occlusion; PcTx1, psalmotoxin 1; SNP, single nucleotide polymorphism; SSD, steady-state desensitization; VCF, voltage-clamp fluorometry.
\end{abstract}

\begin{abstract}
The $\mathrm{pH}$ in the different tissues and organs of our body is kept within tight limits. Local $\mathrm{pH}$ changes occur, however, temporarily under physiological conditions, as for example in synapses during neuronal activity. In pathological situations, such as in ischemia, inflammation, and tumor growth, long-lasting acidification develops. Acid-sensing ion channels (ASICs) are low $\mathrm{pH}$-activated $\mathrm{Na}^{+}$-permeable ion channels that are widely expressed in the central and peripheral nervous systems. ASICs act as $\mathrm{pH}$ sensors, leading to
\end{abstract}


neuronal excitation when the $\mathrm{pH}$ drops. Animal studies have shown that ASICs are involved in several physiological and pathological processes, such as pain sensation, learning, fear sensing, and neurodegeneration after ischemic stroke. ASIC inhibitors could be used as analgesic and anxiolytic drugs, and as drugs for the treatment of ischemic stroke. For these reasons, ASICs have recently attracted increasing attention. Currently, no drugs are clinically used as ASIC modulators. ASICs are however targets of several peptide toxins from animals. Much effort is invested in research studying the function of these channels. We review here the available pharmacological agents acting on ASICs, which include small molecules and animal toxins. We then discuss the current understanding of the molecular mechanisms by which pH controls ASIC activity. Knowledge of the function of ASICs at the molecular level should allow the development of new pharmacological strategies for targeting these promising ion channels.

Keywords: ASIC, ion channel, pharmacology, activation mechanism, desensitization, neuron

(Introduction)

Acid-sensing ion channels (ASICs) are $\mathrm{Na}^{+}$-permeable channels of the nervous system that are transiently activated by extracellular acidification. ASICs form a subfamily of the Epithelial $\mathrm{Na}^{+}$channel $(\mathrm{ENaC}) /$ degenerin channel family. This family comprises among others the amiloride-sensitive $\mathrm{ENaC}$, which is important for reabsorption of $\mathrm{Na}^{+}$in the distal kidney, and contributes to the control of the liquid level on airway epithelia. A second subfamily, the degenerins, forms the channel part of a mechanosensitive complex in neurons of C. elegans, where it contributes to light touch sensation. The cloning of ASICs is indirectly linked to Switzerland, since it was based on the sequence homology of ASICs to $\mathrm{ENaC}$. The $\mathrm{ENaC}$ is a heterotrimeric channel, formed by the homologous $\alpha, \beta$ and $\gamma$ subunits. 
Cloning of the $\alpha$ subunit was achieved by Cecilia Canessa in the laboratory of Bernard Rossier at the Department of Pharmacology of the University of Lausanne [1]. Expression cloning from colon mRNA of salt-depleted rats allowed the cloning of the $\alpha$, and later the $\beta$ and $\gamma \mathrm{ENaC}$ subunits [2]. The cloning of $\mathrm{ENaC}$ led to a very fruitful period of research addressing the function and physiology of $\mathrm{ENaC}$ in cells, in murine models and in human disease [3].

Proton-activated $\mathrm{Na}^{+}$currents of neurons had been measured first in the 1980s [4] and were subsequently described by several laboratories. The identity of the channels mediating these currents remained, however, unknown. Soon after the cloning of ENaC, so-called brain $\mathrm{Na}^{+}$ channels were cloned based on their sequence homology to $\mathrm{ENaC}[5,6]$. These channels showed only very small currents at physiological $\mathrm{pH}$, and their activators were not known. The breakthrough came from the laboratory of Michel Lazdunski, who found that these novel $\mathrm{Na}^{+}$channels were activated by lowering of the extracellular $\mathrm{pH}$ [7]. Initially, the members of this new subfamily of ion channels were considered as the poor cousins of $\mathrm{ENaC}$, since not much was known about their expression and physiological or pathological roles. Very rapidly, several different ASIC subunits were identified, and their expression and function were characterized (rev. in $[8,9])$. Studies with subtype-specific ASIC knockout mice identified diverse roles of ASICs, raising the interest in these channels [10, 11]. Still now, there are, however, no disease-causing mutations of ASICs known, and there are no selective small molecule inhibitors available to confirm their functions observed in animals.

\section{Functional properties of ASICs}

A typical current trace of a voltage-clamp experiment carried out with an ASIC3-expressing cell in the presence of the modulator 2-guanidine-4-methylquinazoline (GMQ, see 3.2) is shown in Fig. 1A. A change of the extracellular $\mathrm{pH}$ from 7.4 to 6 induces a rapidly 
developing inward current. This current is transient and the major part of it does not persist as long as the $\mathrm{pH}$ is kept acidic, because the channels enter rapidly after opening a nonconducting, so-called desensitized state. ASICs can therefore exist in three different functional states, closed, open and desensitized (Fig. 1B). The channels can leave the desensitized state only if the $\mathrm{pH}$ is brought back to a more alkaline value, which allows them to enter the closed state, from which they can be activated again by a subsequent acidification. Four ASIC genes have been identified, and six homologous subunits, ASIC1a, ASIC1b, ASIC2a, ASIC2b, ASIC3 and ASIC4 are known. ASICs can assemble into heteroor homotrimeric channels $[12,13]$, whose properties $(\mathrm{pH}$ dependence, kinetics and ion selectivity) depend on the subunit composition [14]. There is currently no evidence that ASIC4 could form functional channels, and it was shown that co-expression of ASIC4 with other ASIC isoforms decreases their current amplitude [15, 16]. All ASIC subunits except ASIC4 are expressed in sensory neurons of the peripheral nervous system, and ASIC1a, ASIC2a, ASIC2b and ASIC4 are widely expressed in the central nervous system.

Crystal structures of chicken ASIC1a, which shares $90 \%$ sequence homology with human ASIC1a, describe the individual subunits as resembling a hand (extracellular part) and a forearm (transmembrane parts). Accordingly, the individual domains have been given names such as finger, thumb, knuckle, and palm, as shown in Fig. 1C [12]. The most important cavities, to which some pharmacological ligands bind, are the extracellular vestibule located at the pore entry at the level of the "wrist", the central vestibule located above it, and the three "acidic pockets", which open towards the external surface of the channel and are easily solvent-accessible (Fig. 1C, right panel). The channel pore, which contains the channel gates and the selectivity filter, is lined mostly by the transmembrane $\alpha$-helices 2 of the three subunits. Most ASICs are $\sim 10$-fold selective for $\mathrm{Na}^{+}$over $\mathrm{K}^{+}[9,17]$. Homomeric ASIC1a and certain ASIC1a-containing heteromers display in addition a small permeability for $\mathrm{Ca}^{2+}$. 
ASICs are characterized by two types of $\mathrm{pH}$ dependence, 1) the $\mathrm{pH}$ dependence of activation, which can be derived from a plot of the current as function of the $\mathrm{pH}$, and 2) the $\mathrm{pH}$ dependence of steady-state desensitization (SSD), which characterizes the transition from the closed to the desensitized state (Fig. 1D) and thus determines the fraction of channels that are available for opening at a given $\mathrm{pH}$. The midpoint of the $\mathrm{pH}$ dependence of activation is $\sim 6.5$ for ASIC1a and ASIC3, 6.1 for ASIC1b, and 4.5 for ASIC2a [8, 9].
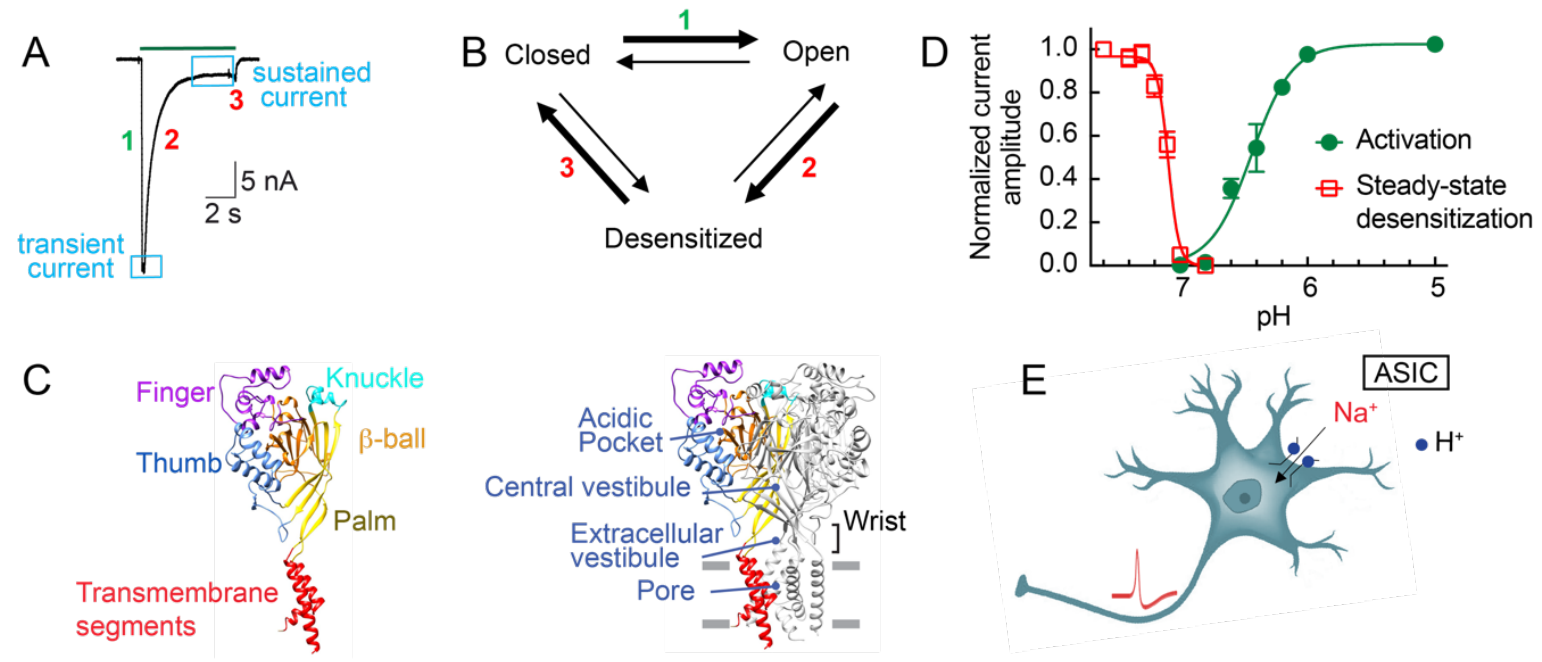

Figure 1. ASIC function and structure. A, Typical ASIC3 current trace, measured in whole-cell voltage-clamp at $-60 \mathrm{mV}$ in the presence of $1 \mathrm{mM}$ GMQ. The extracellular $\mathrm{pH}$ was changed from $\mathrm{pH} 7.4$ to 6 for the time indicated by the horizontal bar. B, Kinetic model of ASIC activity. The numbers in A and B indicate the transitions between the states, as $1=$ opening, 2 = desensitization, and $3=$ recovery from desensitization. C, Structural model of ASICla based on the crystal structure of the channel opened by mit-toxin [18]. Left, single subunit, with coloring of the domains. Right, ASICla trimer of different vestibules and the pore; grey horizontal bars indicate the approximate limits of the membrane. The major part of the channel is extracellular. The acidic pocket constitutes a cavity composed of the thumb, finger and $\beta$-ball domains of one subunit, and the palm domain of an adjacent subunit. Note that one ASIC trimer contains three acidic pockets, one of which is indicated. $\mathrm{D}, \mathrm{pH}$ dependence of steady-state desensitization (SSD, corresponding to the closed $\rightarrow$ desensitized transition, red) and activation (green), shown for ASIC1a. For SSD, the cells were exposed to the indicated conditioning $\mathrm{pH}$ during $60 \mathrm{~s}$, before measurement of the fraction of nondesensitized channels by an acidification to $\mathrm{pH} 6$. For activation, the normalized current amplitude is represented as a function of the stimulating $\mathrm{pH}$. E, Illustration of ASIC 
activation leading to $\mathrm{Na}^{+}$entry, membrane depolarization and induction of an action potential in a neuron.

While ASIC1a- and ASIC1b currents are only transient, the acid-induced currents of ASIC3 and of some heteromeric ASICs do not completely desensitize, leading to an additional sustained current (see Fig. 1A), which is generally of much smaller amplitude than the transient current. This sustained current may mediate slower, and long-lasting effects of sustained $\mathrm{pH}$ changes.

\section{Physiological and pathological roles of ASICs}

Rapid local acidification occurs in synapses during neuronal activity, whereas slower and sustained tissue acidification develops in ischemia, in inflammation, and in tumors [19]. Since ASICs are $\mathrm{Na}^{+}$-permeable, their activation in such situations depolarizes the neuronal membrane and leads to an excitation of the neuron [20,21] (Fig. 1E). Table 1 lists the most important roles of ASICs, and indicates the evidence for these roles. Fear and anxiety, synaptic plasticity and learning, as well as epilepsy, are most likely accompanied by rapid $\mathrm{pH}$ changes in synapses. The other roles of ASICs are associated with situations that are accompanied by slower and sustained tissue acidification. It is currently not well understood how ASICs, which have a mostly transient activity, can be efficient sensors for long-lasting $\mathrm{pH}$ changes. It has been hypothesized that currently unknown modulators modify the ASIC function to enable prolonged activity [22]. ASICs can also act as mechanosensors, as illustrated by their role in the intestine (Table 1). Note also that ASICs are involved in pain perception at two levels, in peripheral sensory neurons, as well as in signal processing in the CNS. The roles of ASICs described in Table 1 are based mostly on results with rodents. For more exhaustive recent discussions, see [8, 9, 19]. Studies on the roles of ASICs in humans are very sparse, to a large extent because potent and selective inhibitors are not available. 
Table 1. Physiological and pathological roles of ASICs

\begin{tabular}{|c|c|}
\hline $\begin{array}{l}\text { Clinical } \\
\text { manifestation }\end{array}$ & Involvement of ASICs \\
\hline Fear and anxiety & $\begin{array}{l}\text { ASIC1a }{ }^{(-/-)} \text {mice showed deficits in fear conditioning and fear } \\
\text { behaviors }[8,23,24] \\
\text { Overexpression of ASIC1a increased fear-related behavior [25] } \\
\text { Increased } \mathrm{CO}_{2} \text { levels induced fear behavior that depended on the } \\
\text { expression of ASIC1a in the amygdala [26] } \\
\text { SNPs of the non-coding region of ASIC1a in humans are } \\
\text { associated with panic disorder and fear-related reactions [27] }\end{array}$ \\
\hline Ischemic stroke & $\begin{array}{l}\text { Pharmacological inhibition or genetic deletion of ASIC1a } \\
\text { strongly reduced the infarct volume in the MCAO model [28] } \\
\text { Increasing the ASIC1a activity worsened the neuronal injury in } \\
\text { the MCAO model [29] }\end{array}$ \\
\hline $\begin{array}{l}\text { Synaptic plasticity and } \\
\text { learning }\end{array}$ & $\begin{array}{l}\text { Genetic deletion of ASIC1a disrupted LTP in synapses of } \\
\text { different regions of the brain [30-32] } \\
\text { ASIC1a }{ }^{(-/)} \text {mice showed mild learning deficits [30] }\end{array}$ \\
\hline Pain sensation & $\begin{array}{l}\text { Specific knockdown of ASIC3 prevented inflammation-induced } \\
\text { heat hyperalgesia in rats [33] } \\
\text { Local injection in the mouse paw of the ASIC } 3 \text { activator GMQ } \\
\text { [34] or of the general ASIC activator mit-toxin [35] induced pain } \\
\text { Inhibition of ASIC1a in the central nervous system by animal } \\
\text { toxins reduced pain }[36,37] \\
\text { Pain induced by injection of acid in the skin of human volunteers } \\
\text { was prevented by amiloride and had the pH dependence of ASICs } \\
{[38,39]}\end{array}$ \\
\hline Migraine & $\begin{array}{l}\text { ASIC-like currents are expressed in dural afferents [40] } \\
\text { Amiloride reduced migraine symptoms in a small clinical trial } \\
\text { [41] } \\
\text { Cutaneous allodynia, which typically occurs with migraine, was } \\
\text { decreased in rats by the ASIC1 inhibitor Mambalgin-1 [42] }\end{array}$ \\
\hline
\end{tabular}




\begin{tabular}{ll}
\hline Intestinal functions & $\begin{array}{l}\text { Selective genetic deletion of ASIC1a, ASIC2 or ASIC3 affected } \\
\text { gastric coordination and emptying, and colonic motility [43, 44] }\end{array}$ \\
\hline Epilepsy & Seizures were inhibited by amiloride in several animal models \\
& {$[45-47]$} \\
& Disrupting the expression of ASIC1a increased, however, the \\
& severity of seizures [48] \\
& An SNP in ASIC1a is associated with temporal lobe epilepsy [49] \\
\hline Genetic disruption of ASIC1a reduced axonal degeneration and \\
the clinical damage in EAE [50] \\
Amiloride was neuroprotective in animals with EAE and humans \\
with multiple sclerosis [51, 52] \\
An SNP in the untranslated region of ASIC2 is associated with \\
multiple sclerosis [53]
\end{tabular}

The data underlying these roles of ASICs are from studies with mice, unless noted differently. EAE, experimental autoimmune encephalomyelitis; GMQ, 2-guanidine-4methylquinazoline; LTP, long-term potentiation; MCAO, middle cerebral artery occlusion; SNP, single nucleotide polymorphism.

\section{Pharmacology of ASICs}

The subunits of ASICs contain many acidic residues, which probably attract the cations that are transported by the channel. Upon extracellular acidification, protonation of some of these residues likely contributes to the activation of ASICs. In addition, many of the known ASICtargeting modulators contain amine or guanidine groups or Arg residues that have an affinity for the acidic groups. This concerns the small molecule inhibitors amiloride and diminazene, as well as neuropeptides that contain an Arg-Phe-amide motive, and several animal toxins that are rich in basic residues. Some of them may bind to the residues that are involved in $\mathrm{H}^{+}-$ dependent ASIC activation. The consequences of the binding of a bigger ligand are, however, likely to be different from those of protonation. 


\subsection{Small molecule inhibitors}

Amiloride (Fig. 2A) blocks the $\mathrm{ENaC}$ pore with an $\mathrm{IC}_{50}$ of $0.1 \mu \mathrm{M}$ and is clinically used as $\mathrm{K}^{+}$-sparing diuretic [54]. It has a lower potency on ASICs $\left(\mathrm{IC}_{50}\right.$ of $10-100 \mu \mathrm{M}$, depending on subtype), where it binds within the channel pore, and inhibits the transient, but not the sustained current component $[9,55]$. Amiloride has been used as ASIC inhibitor in experimental studies in humans $[38,41,52]$. Not surprisingly, at doses that suppress ASIC activity, it inhibits $\mathrm{ENaC}$, and, in addition, other ion channels and several transporters [56]. Diminazene (Fig. 2A), an antiprotozoal drug used in animals, was shown with other diarylamidines to inhibit ASICs at low micromolar concentrations [57]. Diminazene acts as a pore blocker; part of its inhibitory action may also be due to binding to the central vestibule [58-60]. Several non-steroidal anti-inflammatory drugs were reported to inhibit ASIC currents with $\mathrm{IC}_{50}$ values up to $\mathrm{mM}$ concentrations [61] that are not reached during therapy with these drugs. Interestingly, aspirin, diclofenac and ibuprofen were shown to prevent inflammation-induced upregulation of ASIC mRNA in sensory neurons at therapeutic doses [61]. Several small molecule inhibitors of ASICs have been characterized but were not further developed. For current reviews on ASIC pharmacology, see [9, 62, 63].
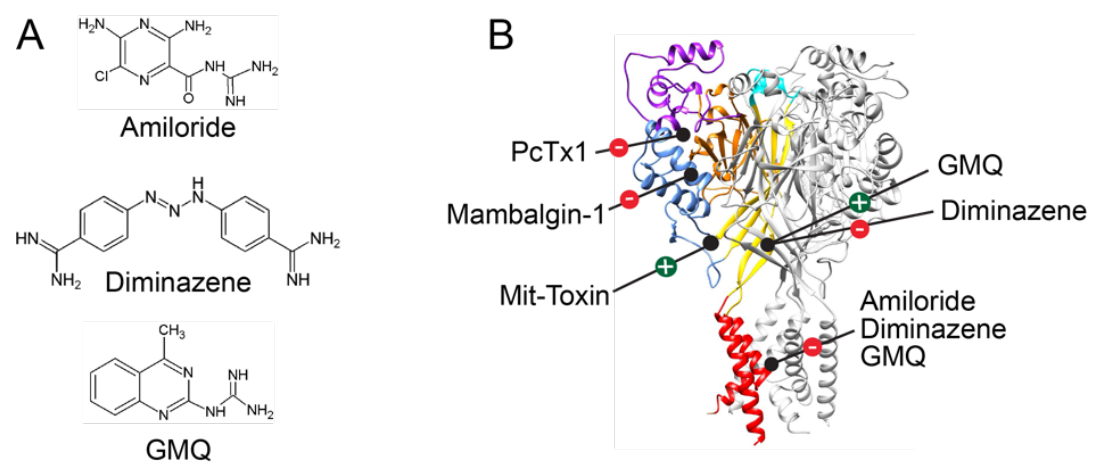

Figure 2. Molecules targeting ASICs. A, Structures of amiloride, diminazene and GMQ. B, Structural model of ASICla (see Fig. legend 1), and illustration of the sites of action of ASIC modulators. Inhibitory actions are indicated by a "_" sign, activation and potentiation by “+”". 


\subsection{ASIC modulators}

ASICs are targets of many endogenous and exogenous modulators $[8,9]$. Among the latter, the compound GMQ (Fig. 2A) has intrigued many researchers, because it was the first nonproton activator of ASICs, and because it drew attention to the central vestibule as potential drug binding site. Initially, GMQ was shown to activate selectively ASIC3 at $\mathrm{pH} 7.4$, and to induce pain in mice in an ASIC3-dependent manner [34]. Based on in silico docking, mutagenesis and functional analysis, the central vestibule was identified as critical site for its functional effects [64]. We reported that GMQ affects the $\mathrm{pH}$ dependence of all studied ASICs by shifting the $\mathrm{pH}$ dependence of SSD to more acidic values. Its effects on activation depend, however, on the subunit composition [55]. GMQ induces an alkaline shift of the $\mathrm{pH}$ dependence of activation in ASIC3, leading to channel activation at $\mathrm{pH} 7.4$; in ASIC1a, this shift goes in the opposite direction and decreases the channel activity [55]. Besides shifting the $\mathrm{pH}$ dependence, GMQ blocks the ASIC pore at higher concentrations [55]. This compound has been useful in the study of gating mechanisms of ASICs, and in the confirmation of the role of ASIC3 in pain sensation (Table 1). An important drawback of GMQ is its low potency on ASICs $\left(\mathrm{EC}_{50}=\sim 3 \mathrm{mM}\right)$.

We have recently synthesized and tested a number of GMQ derivatives, and identified certain guanidinopyridines with a 20-fold increased potency for the pore blocking effect [65]. ASICs containing the subunits $1 \mathrm{a}, 1 \mathrm{~b}$ or 3 are modulated by neuropeptides containing an ArgPhe-amide motive [66]. These shift the $\mathrm{pH}$ dependence of SSD to more acidic values and slow the ASIC current decay, which increases the duration of ASIC activity and may enhance the ASIC response to sustained $\mathrm{pH}$ changes. Modulation by neuropeptides increases indeed pain sensation [67] and cell death after prolonged acidosis [68]. Interestingly, the endogenous opioid peptide Big dynorphin was shown to have similar effects as Arg-Phe-amide peptides 
on ASICs, with an $\mathrm{EC}_{50}$ of as low as $\sim 30 \mathrm{nM}$ [68]. It is therefore likely that dynorphin and structurally related peptides may modulate ASICs under physiological conditions.

\subsection{ASIC modulating toxins}

Several peptide toxins bind to ASICs with nanomolar affinity. The most important toxins are psalmotoxin1 (PcTx1) of the spider Psalmopoeus cambridgei [69], APETx2 from the sea anemone Anthopleura elegantissima [70], mit-toxin of the Texas coral snake (Micrurus tener tener) [35], and mambalgin from the black mamba (Dendroaspis polyepis polyepis) [37]. Since these toxins are peptides of $\geq 40$ amino acid residues, their binding sites on ASICs must be well exposed. Indeed, PcTx1 and Mambalgin were both shown to bind to the thumb $\alpha$-helix 5 of the acidic pocket $[71,72]$, with PcTx1 reaching in addition into the acidic pocket $[73,74]$. The larger mit-toxin binds to the surface of the thumb, palm and wrist [18]. These toxins can be classified as inhibitors (PcTx1, APETx2, Mambalgin) and activators (mittoxin). The inhibitors show ASIC subtype specificity, with PcTx1 inhibiting ASIC1a, Mambalgin ASIC1a, ASIC1b and ASIC1a-containing heteromers, and APETx2 inhibiting homo- and heteromeric ASIC3-containing channels $[9,75]$. APETx2 inhibits also voltagegated $\mathrm{Na}^{+}$channels $[76,77]$. Mechanistically, these toxins act as gating modifiers. PcTx1 shifts the pH dependence of SSD (Fig. 1D) to more alkaline values. Consequently, ASIC1a is desensitized at $\mathrm{pH} 7.4$, and can not be opened when the $\mathrm{pH}$ drops [78]. Mambalgin-1 was shown to shift the $\mathrm{pH}$ dependence of activation (Fig. 1D) of ASIC1a to more acidic values; therefore, stronger acidification is needed to open the ASIC in the presence of the toxin [37]. The molecular mechanism of ASIC activation by mit-toxin is not known. It was, however, shown that ASIC2a currents are increased in the presence of mit-toxin due to an alkaline shift of the $\mathrm{pH}$ dependence of activation [35]. 
Due to their high potency and their ASIC subtype specificity, these toxins were instrumental in defining the physiological and pathological roles of ASICs (Table 1). Purification of toxinASIC complexes allowed the elucidation of the 3D structure of ASIC1a in the open state [18, 73, 74]. Finally, experiments with PcTx1 and Mambalgin indicated that the acidic pocket codetermines the ASIC $\mathrm{pH}$ dependence.

Taken together, ASICs are potential drug targets of high interest. To date, no potent and selective small molecule modulators of ASICs are available. The crucial role of the toxins in elucidating ASIC functions in animal models illustrates how important the availability of ASIC-selective small molecule modulators would be to confirm the predicted roles of ASICs in humans. A molecular understanding of the function of ASICs would certainly enhance the chances to identify such modulators.

\section{Activation mechanism of ASICs}

It is generally thought that proton binding to the extracellular domain initiates conformational changes that are transmitted through the wrist to the transmembrane domains to promote pore opening. To understand the activation mechanism of ASICs, it would therefore be important to identify the protonation sites and to know the conformational changes that transmit the protonation signal from these sites to the channel gate. We will discuss here 1) information from crystal structures, 2) investigation of the protonation sites, and 3) findings on conformational changes from diverse approaches.

\subsection{ASIC structures in the closed, open and desensitized state}

Superposition of the closed, open and desensitized structures suggests a conserved structural scaffold defined by the upper palm and knuckle domains (Fig. 1C), which adopt the same 
conformation in the three states $[18,79]$. The main differences between the closed and open states reside in the conformation of the acidic pocket and in the geometry of the transmembrane domains (Fig. 3). The transition from the closed to the open state is accompanied by the collapse of the acidic pocket from an extended conformation. It has been suggested that low $\mathrm{pH}$ drives this collapse and leads to structural rearrangements through the extracellular domain, culminating in the expansion of the wrist region and in the widening of the pore $[12,79]$. Continued exposure to protons leads to desensitization of the channel by inducing changes allowing the wrist and pore to relax back into a narrower, non-conducting conformation that resembles the closed state (Fig. 3).

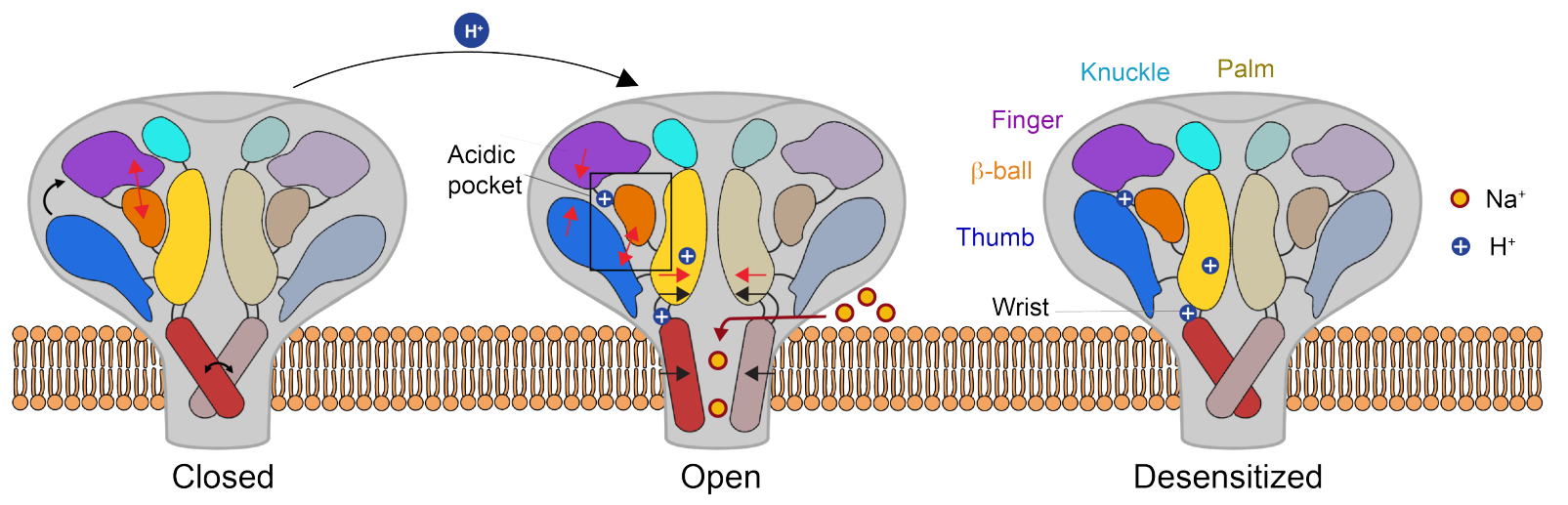

Figure 3. Hypothetical conformational changes during ASIC gating. Cartoons of an ASIC trimer, showing two of the three subunits, in the closed, open and desensitized state are depicted. In the closed state, the acidic pocket adopts an extended conformation with the thumb pushed away from the $\beta$-ball and finger domains. The transmembrane domains assume a constricted conformation that shuts the gate and prevents the passage of ions. Protonation of several ASIC domains (highlighted with blue circles) induces the collapse of the acidic pocket and conformational changes in the extracellular and transmembrane domains lead to the opening of the pore. The permeation of $\mathrm{Na}^{+}$ions through the open channel is illustrated. Channel desensitization is accompanied by substantial reorganization of the acidic pocket, and a shift of the palm and the transmembrane domains that leads to the closing of the pore. The 
red and black arrows highlight the conformational changes in the subsequent transition (closed state: $\rightarrow$ opening; open state: $\rightarrow$ desensitization). Black arrows, derived from the comparison of crystal structures; red arrows, evidence from voltage-clamp fluorometry studies.

\subsection{Protonation sites}

The crystal structures provide snapshots of the architecture as well as the structural relations between domains. They do however not identify the residues that need to be protonated to induce ASIC activation. Different functional approaches have been used to identify potential $\mathrm{pH}$ sensors in the extracellular domain of ASICs. Identification of proton-sensing sites is complicated by the fact that mutation of many residues - even those not directly involved in proton binding - may affect the $\mathrm{pH}$ dependence of ASIC currents. One study exploited the difference in proton sensitivity between ASICs of two evolutionary distant species, the nonproton-sensitive lamprey ASIC1 and rat ASIC1. Mutation of two residues located in the palm domain was sufficient to convert a nonfunctional ASIC1 into a proton-gated channel [80]. The two mutations are located in the part of the palm that is connected to the first transmembrane segment. Changes in this part of the palm are likely involved in the transmission of conformational changes from the ectodomain to the channel pore. Other studies exploited the differences between the $\mathrm{pH}$-sensitive ASIC2a and the $\mathrm{pH}$-insensitive ASIC2b splice variants to conclude that the first 87 residues after the first transmembrane domain are critical for ASIC2 activation by protons $[81,82]$.

The wrist, located at the interface of the palm and the transmembrane segments, contains several His and acidic residues. Simultaneous mutation of several of these potential protonsensing residues led to non-functional channels that were, however, still expressed at the cell surface, suggesting that this region may be critically involved in ASIC proton sensing [83]. Other studies identified residues in the $\beta$-ball and palm domains as key molecular determinants 
of ASIC proton sensitivity $[84,85]$. Liechti and colleagues estimated $\mathrm{p} K_{\mathrm{a}}$ values of Asp, Glu and His residues based on the desensitized ASIC1 a structure and tested the functional relevance of residues with a predicted $\mathrm{p} K_{\mathrm{a}}$ between 7.4 and 5 . Their analysis identified several residues in the palm, thumb and $\beta$-ball domains as putative proton sensors [86].

Because of its high negative electrostatic potential due to the presence of many acidic residues, the acidic pocket represents an excellent candidate for conferring proton sensing to ASICs. Consistent with this property, channels harboring individual or combined neutralizing mutations within this pocket showed reduced proton affinity. However, combined mutation of a large number of titratable residues in the acidic pocket resulted in channels that retained the ability to open in response to acidification, indicating that proton sensing in the acidic pocket influences ASIC $\mathrm{pH}$ dependence, but is not required for ASIC activation [12, 87].

In conclusion, the hypothesis that $\mathrm{pH}$ sensing in the acidic pocket drives ASIC opening, as suggested by the structural work, was not confirmed by functional studies. Rather, ASIC activation depends on protonation of multiple residues located in different channel domains.

\subsection{Evidence for conformational changes during ASIC activity}

Since some of the protonation sites are located quite distantly from the channel pore where the gate resides, it is expected that protonation of these sites allosterically affects the state of the channel gate. Several studies have investigated the structural rearrangements occurring in different channel domains during ASIC activation. Measurements using luminescence resonance energy transfer that measures distances between two sites in a given protein, reported structural rearrangements in the thumb and finger domains of ASIC1a, consistent with a decreased distance between these two domains in the desensitized as compared to closed state [88]. A reduced distance between the thumb and finger domains during ASIC desensitization has also been observed in a study from our laboratory that employed voltage-clamp 
fluorometry (VCF) [87]. This study involved introduction of a Cys residue into a domain of interest, expression of the mutant channel in Xenopous oocytes, and selective attachment of an environmentally sensitive fluorophore to the engineered Cys residue. The fluorescence signal, which reports conformational changes, was then measured simultaneously with the current. This approach allowed a direct correlation between the observed movements at a distinct location within the channel and a specific channel transition [89]. Studies conducted on ASICs using VCF suggest that proton binding induces conformational changes in different ASIC domains. Rapid and slow conformational changes have been detected in the acidic pocket, consistent with a role in both, activation and desensitization (Fig. 3) [87]. Rapid conformational changes have also been observed in the finger and knuckle domains [90, 91].

To derive information on conformational changes, several studies have measured the accessibility of engineered Cys residues to Cys-specific methanethiosulfonate reagents. By using this technique, it was shown that the modification rate of engineered Cys residues pointing to the central vestibule by a positively charged methanethiosulfonate reagent was decreased when the reagent was applied to the desensitized channels as compared to application to the closed state, suggesting that the lower palm domains of the three subunits undergo a closing movement during desensitization $[92,93]$.

The introduction of disulfide bonds can constrain inter-domain or inter-subunit distances, and with this prevent conformational changes. The consequences of formation or cleavage of disulfide bonds can be used to investigate the link between local changes in conformation and channel function. This approach has been used to confirm that the collapse of the acidic pocket is required for ASIC activation [79]. Analysis of inter-subunit disulfide bonds at the level of the thumb and palm indicated that the subunits need to move away from each other to allow pore opening [91]. 
Taken together, the crystal structures and functional approaches indicate that upon extracellular acidification, the acidic pocket collapses, and the wrist and pore adopt an extended open conformation, allowing current flow. If the acidification persists, the lower palm and, with it, the wrist and the pore, collapse. With these changes, the channel enters the desensitized state. The conformational changes are driven by protonation in different parts of the ASIC ectodomain.

\section{Conclusion and perspectives}

ASICs have recently attracted great interest because of their involvement in physiological and pathological processes, as revealed by animal studies. However, most of these proposed functions have not been confirmed in humans, probably in part due to the lack of potent and selective ASIC modulators. With the ongoing progress in the elucidation of the molecular mechanisms of ASIC function, and with the availability of new screening approaches, it will hopefully be possible to develop such modulators in the near future. This would allow testing of the relevance of ASICs in human physiology and pathology, and may give rise to new anxiolytic and analgesic drugs, and drugs that reduce neuronal death after ischemic stroke.

\section{Acknowledgements}

Research in the author's laboratory is financed by the Swiss National Science Foundation grant 31003A_172968. We thank Olivier Bignucolo, Zhong Peng and Anand Vaithia for comments on the manuscript.

\section{Conflict of interest}

The authors declare no conflict of interest. 


\section{References}

[1] C.M. Canessa, J.-D. Horisberger, B.C. Rossier, Epithelial sodium channel related to proteins involved in neurodegeneration, Nature 361 (1993) 467-470.

[2] C.M. Canessa, L. Schild, G. Buell, B. Thorens, I. Gautschi, J.D. Horisberger, B.C. Rossier, Amiloride-sensitive epithelial $\mathrm{Na}+$ channel is made of three homologous subunits, Nature 367(6462) (1994) 463-7.

[3] B.C. Rossier, Epithelial sodium channel (ENaC) and the control of blood pressure, Curr. Opin. Pharmacol 15C (2014) 33-46.

[4] O.A. Krishtal, V.I. Pidoplichko, A receptor for protons in the membrane of sensory neurons may participate in nociception, Neurosci. 6 (1981) 2599-2601.

[5] J. Garcia-Anoveros, B. Derfler, J. Nevillegolden, B.T. Hyman, D.P. Corey, BNaC1 and $\mathrm{BNaC} 2$ constitute at new family of human neuronal sodium channels related to degenerins and epithelial sodium channels, Proc. Natl. Acad. Sci. USA 94(4) (1997) $1459-1464$.

[6] M.P. Price, P.M. Snyder, M.J. Welsh, Cloning and expression of a novel human brain $\mathrm{Na}^{+}$ channel, J. Biol. Chem. 271 (1996) 7879-7882.

[7] R. Waldmann, G. Champigny, F. Bassilana, C. Heurteaux, M. Lazdunski, A proton-gated cation channel involved in acid-sensing, Nature 386(6621) (1997) 173-177.

[8] J.A. Wemmie, R.J. Taugher, C.J. Kreple, Acid-sensing ion channels in pain and disease, Nature Rev. Neurosci. 14(7) (2013) 461-71.

[9] S. Kellenberger, L. Schild, International Union of Basic and Clinical Pharmacology. XCI. Structure, Function, and Pharmacology of Acid-Sensing Ion Channels and the Epithelial $\mathrm{Na}^{+}$Channel, Pharmacol Rev 67(1) (2015) 1-35.

[10] M.P. Price, G.R. Lewin, S.L. Mcllwrath, C. Cheng, J. Xie, P.A. Heppenstall, C.L. Stucky, A.G. Mannsfeldt, T.J. Brennan, H.A. Drummond, J. Qiao, C.J. Benson, D.E. 
Tarr, R.F. Hrstka, B. Yang, R.A. Williamson, M.J. Welsh, The mammalian sodium channel BNC1 is required for normal touch sensation, Nature 407 (2000) 1007-1011.

[11] M.P. Price, S.L. McIlwrath, J. Xie, C. Cheng, J. Qiao, D.E. Tarr, K.A. Sluka, T.J. Brennan, G.R. Lewin, M.J. Welsh, The DRASIC cation channel contributes to the detection of cutaneous touch and acid stimuli in mice, Neuron 32(6) (2001) 1071-83.

[12] J. Jasti, H. Furukawa, E.B. Gonzales, E. Gouaux, Structure of acid-sensing ion channel 1 at 1.9 A resolution and low pH, Nature 449(7160) (2007) 316-23.

[13] T. Bartoi, K. Augustinowski, G. Polleichtner, S. Grunder, M.H. Ulbrich, Acid-sensing ion channel (ASIC) 1a/2a heteromers have a flexible 2:1/1:2 stoichiometry, Proc Natl Acad Sci U S A 111(22) (2014) 8281-6.

[14] M. Hesselager, D.B. Timmermann, P.K. Ahring, pH dependency and desensitization kinetics of heterologously expressed combinations of acid-sensing ion channel subunits, J. Biol. Chem. 279(12) (2004) 11006-11015.

[15] E. Donier, F. Rugiero, C. Jacob, J.N. Wood, Regulation of ASIC activity by ASIC4-new insights into ASIC channel function revealed by a yeast two-hybrid assay, Eur J Neurosci 28(1) (2008) 74-86.

[16] S.-H. Lin, Y.-C. Chien, W.-W. Chiang, Y.-Z. Liu, C.-C. Lien, C.-C. Chen, Genetic mapping of ASIC4 and contrasting phenotype to ASIC1a in modulating innate fear and anxiety, Eur. J. Neurosci. 41(12) (2015) 1553-1568.

[17] L. Yang, L.G. Palmer, Ion conduction and selectivity in acid-sensing ion channel 1, J Gen Physiol 144(3) (2014) 245-255.

[18] I. Baconguis, C.J. Bohlen, A. Goehring, D. Julius, E. Gouaux, X-ray structure of Acidsensing ion channel 1-snake toxin complex reveals open state of a $\mathrm{Na}^{+}$-selective channel, Cell 156(4) (2014) 717-29. 
[19] E. Boscardin, O. Alijevic, E. Hummler, S. Frateschi, S. Kellenberger, The function and regulation of acid-sensing ion channels (ASICs) and the epithelial $\mathrm{Na}(+)$ channel (ENaC): IUPHAR Review 19, Br J Pharmacol 173(18) (2016) 2671-701.

[20] E. Deval, A. Baron, E. Lingueglia, H. Mazarguil, J.M. Zajac, M. Lazdunski, Effects of neuropeptide SF and related peptides on acid sensing ion channel 3 and sensory neuron excitability, Neuropharmacol. 44(5) (2003) 662-71.

[21] M. Vukicevic, S. Kellenberger, Modulatory effects of acid-sensing ion channels on action potential generation in hippocampal neurons, Am J Physiol Cell Physiol 287(3) (2004) C682-90.

[22] S. Marra, R. Ferru-Clement, V. Breuil, A. Delaunay, M. Christin, V. Friend, S. Sebille, C. Cognard, T. Ferreira, C. Roux, L. Euller-Ziegler, J. Noel, E. Lingueglia, E. Deval, Non-acidic activation of pain-related Acid-Sensing Ion Channel 3 by lipids, EMBO J 35(4) (2016) 414-28.

[23] J.A. Wemmie, C.C. Askwith, E. Lamani, M.D. Cassell, J.H. Freeman, Jr., M.J. Welsh, Acid-sensing ion channel 1 is localized in brain regions with high synaptic density and contributes to fear conditioning, J Neurosci 23(13) (2003) 5496-502.

[24] M.W. Coryell, A.M. Wunsch, J.M. Haenfler, J.E. Allen, J.L. McBride, B.L. Davidson, J.A. Wemmie, Restoring Acid-Sensing Ion Channel-1a in the Amygdala of Knock-Out Mice Rescues Fear Memory But Not Unconditioned Fear Responses, J. Neurosci. 28(51) (2008) 13738-13741.

[25] J.A. Wemmie, M.W. Coryell, C.C. Askwith, E. Lamani, A.S. Leonard, C.D. Sigmund, M.J. Welsh, Overexpression of acid-sensing ion channel 1a in transgenic mice increases acquired fear-related behavior, Proc Natl Acad Sci U S A 101(10) (2004) 3621-6.

[26] A.E. Ziemann, J.E. Allen, N.S. Dahdaleh, Drebot, II, M.W. Coryell, A.M. Wunsch, C.M. Lynch, F.M. Faraci, M.A. Howard, 3rd, M.J. Welsh, J.A. Wemmie, The amygdala 
is a chemosensor that detects carbon dioxide and acidosis to elicit fear behavior, Cell 139(5) (2009) 1012-21.

[27] J.W. Smoller, P.J. Gallagher, L.E. Duncan, L.M. McGrath, S.A. Haddad, A.J. Holmes, A.B. Wolf, S. Hilker, S.R. Block, S. Weill, S. Young, E.Y. Choi, J.F. Rosenbaum, J. Biederman, S.V. Faraone, J.L. Roffman, G.G. Manfro, C. Blaya, D.R. Hirshfeld-Becker, M.B. Stein, M. Van Ameringen, D.F. Tolin, M.W. Otto, M.H. Pollack, N.M. Simon, R.L. Buckner, D. Ongur, B.M. Cohen, The Human Ortholog of Acid-Sensing Ion Channel Gene ASIC1a Is Associated with Panic Disorder and Amygdala Structure and Function, Biological psychiatry 10.1016/j.biopsych.2013.12.018 (2014).

[28] Z.G. Xiong, X.M. Zhu, X.P. Chu, M. Minami, J. Hey, W.L. Wei, J.F. MacDonald, J.A. Wemmie, M.P. Price, M.J. Welsh, R.P. Simon, Neuroprotection in ischemia: Blocking calcium-permeable acid- sensing ion channels, Cell 118(6) (2004) 687-698.

[29] B. Duan, Y.Z. Wang, T. Yang, X.P. Chu, Y. Yu, Y. Huang, H. Cao, J. Hansen, R.P. Simon, M.X. Zhu, Z.G. Xiong, T.L. Xu, Extracellular spermine exacerbates ischemic neuronal injury through sensitization of ASIC1a channels to extracellular acidosis, $\mathrm{J}$ Neurosci 31(6) (2011) 2101-12.

[30] J.A. Wemmie, J. Chen, C.C. Askwith, A.M. Hruska-Hageman, M.P. Price, B.C. Nolan, P.G. Yoder, E. Lamani, T. Hoshi, J.H. Freeman, Jr., M.J. Welsh, The acid-activated ion channel ASIC contributes to synaptic plasticity, learning, and memory, Neuron 34(3) (2002) 463-77.

[31] J. Du, L.R. Reznikov, M.P. Price, X.M. Zha, Y. Lu, T.O. Moninger, J.A. Wemmie, M.J. Welsh, Protons are a neurotransmitter that regulates synaptic plasticity in the lateral amygdala, Proc Natl Acad Sci U S A 111(24) (2014) 8961-6. 
[32] M.G. Liu, H.S. Li, W.G. Li, Y.J. Wu, S.N. Deng, C. Huang, O. Maximyuk, V. Sukach, O. Krishtal, M.X. Zhu, T.L. Xu, Acid-sensing ion channel 1a contributes to hippocampal LTP inducibility through multiple mechanisms, Sci Rep 6 (2016).

[33] E. Deval, J. Noel, N. Lay, A. Alloui, S. Diochot, V. Friend, M. Jodar, M. Lazdunski, E. Lingueglia, ASIC3, a sensor of acidic and primary inflammatory pain, EMBO J 27(22) (2008) 3047-55.

[34] Y. Yu, Z. Chen, W.G. Li, H. Cao, E.G. Feng, F. Yu, H. Liu, H. Jiang, T.L. Xu, A nonproton ligand sensor in the acid-sensing ion channel, Neuron 68(1) (2010) 61-72.

[35] C.J. Bohlen, A.T. Chesler, R. Sharif-Naeini, K.F. Medzihradszky, S. Zhou, D. King, E.E. Sanchez, A.L. Burlingame, A.I. Basbaum, D. Julius, A heteromeric Texas coral snake toxin targets acid-sensing ion channels to produce pain, Nature 479(7373) (2011) 410-4.

[36] M. Mazzuca, C. Heurteaux, A. Alloui, S. Diochot, A. Baron, N. Voilley, N. Blondeau, P. Escoubas, A. Gelot, A. Cupo, A. Zimmer, A.M. Zimmer, A. Eschalier, M. Lazdunski, A tarantula peptide against pain via ASIC1a channels and opioid mechanisms, Nat Neurosci 10(8) (2007) 943-5.

[37] S. Diochot, A. Baron, M. Salinas, D. Douguet, S. Scarzello, A.S. Dabert-Gay, D. Debayle, V. Friend, A. Alloui, M. Lazdunski, E. Lingueglia, Black mamba venom peptides target acid-sensing ion channels to abolish pain, Nature 490(7421) (2012) 5525.

[38] S. Ugawa, T. Ueda, Y. Ishida, M. Nishigaki, Y. Shibata, S. Shimada, Amilorideblockable acid-sensing ion channels are leading acid sensors expressed in human nociceptors, J. Clin. Invest. 110(8) (2002) 1185-1190.

[39] N.G. Jones, R. Slater, H. Cadiou, P. McNaughton, S.B. McMahon, Acid-Induced Pain and Its Modulation in Humans, J. Neurosci. 24(48) (2004) 10974-10979. 
[40] J. Yan, R.M. Edelmayer, X. Wei, M. De Felice, F. Porreca, G. Dussor, Dural afferents express acid-sensing ion channels: a role for decreased meningeal $\mathrm{pH}$ in migraine headache, Pain 152(1) (2011) 106-13.

[41] P.R. Holland, S. Akerman, A.P. Andreou, N. Karsan, J.A. Wemmie, P.J. Goadsby, Acidsensing ion channel 1: a novel therapeutic target for migraine with aura, Ann. Neurolo. 72(4) (2012) 559-63.

[42] C. Verkest, E. Piquet, S. Diochot, M. Dauvois, M. Lanteri-Minet, E. Lingueglia, A. Baron, Effects of systemic inhibitors of acid-sensing ion channels 1 (ASIC1) against acute and chronic mechanical allodynia in a rodent model of migraine, Brit. J. Pharmacol. 175(21) (2018) 4154-4166.

[43] R.C. Jones, 3rd, L. Xu, G.F. Gebhart, The mechanosensitivity of mouse colon afferent fibers and their sensitization by inflammatory mediators require transient receptor potential vanilloid 1 and acid-sensing ion channel 3, J Neurosci 25(47) (2005) 10981-9.

[44] A.J. Page, S.M. Brierley, C.M. Martin, M.P. Price, E. Symonds, R. Butler, J.A. Wemmie, L.A. Blackshaw, Different contributions of ASIC channels 1a, 2, and 3 in gastrointestinal mechanosensory function, Gut 54(10) (2005) 1408-15.

[45] A. Ali, K.P. Pillai, F.J. Ahmad, Y. Dua, D. Vohora, Anticonvulsant effect of amiloride in pentetrazole-induced status epilepticus in mice, Pharmacol. Rep. : PR 58(2) (2006) 242-5.

[46] J.J. Luszczki, K.M. Sawicka, J. Kozinska, M. Dudra-Jastrzebska, S.J. Czuczwar, Amiloride enhances the anticonvulsant action of various antiepileptic drugs in the mouse maximal electroshock seizure model, J Neural Transm 116(1) (2009) 57-66.

[47] P. N'Gouemo, Amiloride delays the onset of pilocarpine-induced seizures in rats, Brain Res 1222 (2008) 230-2. 
[48] A.E. Ziemann, M.K. Schnizler, G.W. Albert, M.A. Severson, M.A. Howard, 3rd, M.J. Welsh, J.A. Wemmie, Seizure termination by acidosis depends on ASIC1a, Nat Neurosci 11(7) (2008) 816-22.

[49] R.J. Lv, J.S. He, Y.H. Fu, Y.Q. Zhang, X.Q. Shao, L.W. Wu, Q. Lu, L.R. Jin, H. Liu, ASIC1a polymorphism is associated with temporal lobe epilepsy, Epilepsy Res. 96(1-2) (2011) 74-80.

[50] M.A. Friese, M.J. Craner, R. Etzensperger, S. Vergo, J.A. Wemmie, M.J. Welsh, A. Vincent, L. Fugger, Acid-sensing ion channel-1 contributes to axonal degeneration in autoimmune inflammation of the central nervous system, Nat Med 13(12) (2007) 1483-9.

[51] S. Vergo, M.J. Craner, R. Etzensperger, K. Attfield, M.A. Friese, J. Newcombe, M. Esiri, L. Fugger, Acid-sensing ion channel 1 is involved in both axonal injury and demyelination in multiple sclerosis and its animal model, Brain 134(Pt 2) (2011) 571-84.

[52] T. Arun, V. Tomassini, E. Sbardella, M.B. de Ruiter, L. Matthews, M.I. Leite, R. Gelineau-Morel, A. Cavey, S. Vergo, M. Craner, L. Fugger, A. Rovira, M. Jenkinson, J. Palace, Targeting ASIC1 in primary progressive multiple sclerosis: evidence of neuroprotection with amiloride, Brain 136(Pt 1) (2013) 106-15.

[53] L. Bernardinelli, S.B. Murgia, P.P. Bitti, L. Foco, R. Ferrai, L. Musu, I. Prokopenko, R. Pastorino, V. Saddi, A. Ticca, M.L. Piras, D.R. Cox, C. Berzuini, Association between the ACCN1 gene and multiple sclerosis in Central East Sardinia, PloS one 2(5) (2007) e480.

[54] H.P. Rang, J.M. Ritter, R.J. Flower, G. Henderson, Rang \& Dale's Pharmacology, eighth edition ed., Elsevier Churchill Livingstone 2016.

[55] O. Alijevic, S. Kellenberger, Subtype-specific modulation of acid-sensing ion channel (ASIC) function by 2-guanidine-4-methylquinazoline, J Biol Chem 287(43) (2012) 36059-70. 
[56] T.R. Kleyman, E.J. Cragoe, Amiloride and Its Analogs as Tools in the Study of IonTransport, J Membrane Biol 105(1) (1988) 1-21.

[57] X. Chen, L. Qiu, M. Li, S. Durrnagel, B.A. Orser, Z.G. Xiong, J.F. MacDonald, Diarylamidines: high potency inhibitors of acid-sensing ion channels, Neuropharmacol. 58(7) (2010) 1045-53.

[58] A. Schmidt, G. Rossetti, S. Joussen, S. Grunder, Diminazene Is a Slow Pore Blocker of Acid-Sensing Ion Channel 1a (ASIC1a), Mol Pharmacol 92(6) (2017) 665-675.

[59] A.J. Krauson, J.G. Rooney, M.D. Carattino, Molecular basis of inhibition of acid sensing ion channel 1A by diminazene, PloS one 13(5) (2018).

[60] J.Y.P. Lee, N.J. Saez, B. Cristofori-Armstrong, R. Anangi, G.F. King, M.T. Smith, L.D. Rash, Inhibition of acid-sensing ion channels by diminazene and APETx2 evoke partial and highly variable antihyperalgesia in a rat model of inflammatory pain, British Journal of Pharmacology 175(12) (2018) 2204-2218.

[61] N. Voilley, J. de Weille, J. Mamet, M. Lazdunski, Nonsteroid anti-inflammatory drugs inhibit both the activity and the inflammation-induced expression of acid-sensing ion channels in nociceptors, J Neurosci 21(20) (2001) 8026-8033.

[62] A. Baron, E. Lingueglia, Pharmacology of acid-sensing ion channels - Physiological and therapeutical perspectives, Neuropharmacology 94 (2015) 19-35.

[63] L.D. Rash, Acid-Sensing Ion Channel Pharmacology, Past, Present, and Future, Adv Pharmacol 79 (2017) 35-66.

[64] Y. Yu, W.G. Li, Z. Chen, H. Cao, H. Yang, H. Jiang, T.L. Xu, Atomic level characterization of the nonproton ligand-sensing domain of ASIC3 channels, J Biol Chem 286(28) (2011) 24996-5006. 
[65] O. Alijevic, H. Hammoud, A. Vaithia, V. Trendafilov, M. Bollenbach, M. Schmitt, F. Bihel, S. Kellenberger, Heteroarylguanidines as Allosteric Modulators of ASIC1a and ASIC3 Channels, ACS Chem. Neurosci. (2018).

[66] J.S. Vick, C.C. Askwith, ASICs and neuropeptides, Neuropharmacol 94 (2015) 36-41.

[67] C. Reimers, C.H. Lee, H. Kalbacher, Y. Tian, C.H. Hung, A. Schmidt, L. Prokop, S. Kauferstein, D. Mebs, C.C. Chen, S. Grunder, Identification of a cono-RFamide from the venom of Conus textile that targets ASIC3 and enhances muscle pain, Proc Natl Acad Sci U S A 114(17) (2017) E3507-E3515.

[68] T.W. Sherwood, C.C. Askwith, Dynorphin opioid peptides enhance acid-sensing ion channel 1a activity and acidosis-induced neuronal death, J Neurosci 29(45) (2009) 14371-80.

[69] P. Escoubas, J.R. DeWeille, A. Lecoq, S. Diochot, R. Waldmann, G. Champigny, D. Moinier, A. Ménez, M. Lazdunski, Isolation of a tarantula toxin specific for a class of proton-gated $\mathrm{Na}^{+}$channels, J. Biol. Chem. 275 (2000) 25116-25121.

[70] S. Diochot, A. Baron, L.D. Rash, E. Deval, P. Escoubas, S. Scarzello, M. Salinas, M. Lazdunski, A new sea anemone peptide, APETx2, inhibits ASIC3, a major acid-sensitive channel in sensory neurons, EMBO J. 23(7) (2004) 1516-1525.

[71] C.I. Schroeder, L.D. Rash, X. Vila-Farres, K.J. Rosengren, M. Mobli, G.F. King, P.F. Alewood, D.J. Craik, T. Durek, Chemical synthesis, 3D structure, and ASIC binding site of the toxin mambalgin-2, Angew Chem Int Ed Engl 53(4) (2014) 1017-20.

[72] D.M. Sun, Y. Yu, X.B. Xue, M. Pan, M. Wen, S.Y. Li, Q. Qu, X.R. Li, L.H. Zhang, X.M. Li, L. Liu, M.J. Yang, C.L. Tian, Cryo-EM structure of the ASIC1 a-mambalgin-1 complex reveals that the peptide toxin mambalgin-1 inhibits acid-sensing ion channels through an unusual allosteric effect, Cell Discov 4 (2018). 
[73] I. Baconguis, E. Gouaux, Structural plasticity and dynamic selectivity of acid-sensing ion channel-spider toxin complexes, Nature 489(7416) (2012) 400-405.

[74] R.J. Dawson, J. Benz, P. Stohler, T. Tetaz, C. Joseph, S. Huber, G. Schmid, D. Hugin, P. Pflimlin, G. Trube, M.G. Rudolph, M. Hennig, A. Ruf, Structure of the Acid-sensing ion channel 1 in complex with the gating modifier Psalmotoxin 1, Nature commun. 3 (2012) 936.

[75] A. Baron, S. Diochot, M. Salinas, E. Deval, J. Noel, E. Lingueglia, Venom toxins in the exploration of molecular, physiological and pathophysiological functions of acid-sensing ion channels, Toxicon 75 (2013) 187-204.

[76] M.G. Blanchard, L.D. Rash, S. Kellenberger, Inhibition of voltage-gated $\mathrm{Na}^{+}$currents in sensory neurones by the sea anemone toxin APETx2, Br J Pharmacol 165(7) (2012) 2167-77.

[77] S. Peigneur, L. Beress, C. Moller, F. Mari, W.G. Forssmann, J. Tytgat, A natural point mutation changes both target selectivity and mechanism of action of sea anemone toxins, FASEB J 26(12) (2012) 5141-51.

[78] X. Chen, H. Kalbacher, S. Grunder, The Tarantula Toxin Psalmotoxin 1 Inhibits Acidsensing Ion Channel (ASIC) 1a by Increasing Its Apparent $\mathrm{H}^{+}$Affinity, J. Gen. Physiol. $126(1)(2005) 71-79$.

[79] N. Yoder, C. Yoshioka, E. Gouaux, Gating mechanisms of acid-sensing ion channels, Nature 555(7696) (2018) 397-401.

[80] T. Li, Y. Yang, C.M. Canessa, Two residues in the extracellular domain convert a nonfunctional ASIC1 into a proton-activated channel, Am J Physiol Cell Physiol 299(1) (2010) C66-73.

[81] E.S. Smith, X. Zhang, H. Cadiou, P.A. McNaughton, Proton binding sites involved in the activation of acid-sensing ion channel ASIC2a, Neurosci Lett 426(1) (2007) 12-7. 
[82] L.N. Schuhmacher, S. Srivats, E.S. Smith, Structural domains underlying the activation of acid-sensing ion channel 2a, Mol Pharmacol 87(4) (2015) 561-71.

[83] M. Paukert, X. Chen, G. Polleichtner, H. Schindelin, S. Grunder, Candidate amino acids involved in $\mathrm{H}^{+}$gating of acid-sensing ion channel 1a, J. Biol. Chem. 283(1) (2008) 57281.

[84] A.J. Krauson, A.C. Rued, M.D. Carattino, Independent contribution of extracellular proton binding sites to ASICla activation, J Biol Chem 288(48) (2013) 34375-83.

[85] T. Lynagh, Y. Mikhaleva, J.M. Colding, J.C. Glover, S.A. Pless, Acid-sensing ion channels emerged over 600 Mya and are conserved throughout the deuterostomes, Proc Natl Acad Sci U S A 115(33) (2018) 8430-8435.

[86] L.A. Liechti, S. Berneche, B. Bargeton, J. Iwaszkiewicz, S. Roy, O. Michielin, S. Kellenberger, A combined computational and functional approach identifies new residues involved in pH-dependent gating of ASIC1a, J Biol Chem 285(21) (2010) 16315-29.

[87] S. Vullo, G. Bonifacio, S. Roy, N. Johner, S. Berneche, S. Kellenberger, Conformational dynamics and role of the acidic pocket in ASIC pH-dependent gating, Proc Natl Acad Sci U S A 114(14) (2017) 3768-3773.

[88] S.S. Ramaswamy, D.M. MacLean, A.A. Gorfe, V. Jayaraman, Proton-mediated Conformational Changes in an Acid-sensing Ion Channel, J Biol Chem 288(50) (2013) 35896-35903.

[89] S. Talwar, J.W. Lynch, Investigating ion channel conformational changes using voltage clamp fluorometry, Neuropharmacology 98 (2015) 3-12.

[90] G. Bonifacio, C.I. Lelli, S. Kellenberger, Protonation controls ASIC1a activity via coordinated movements in multiple domains, J Gen Physiol 143(1) (2014) 105-18. 
[91] K. Gwiazda, G. Bonifacio, S. Vullo, S. Kellenberger, Extracellular Subunit Interactions Control Transitions between Functional States of Acid-sensing Ion Channel 1a, J Biol Chem 290(29) (2015) 17956-66.

[92] S. Roy, C. Boiteux, O. Alijevic, C. Liang, S. Berneche, S. Kellenberger, Molecular determinants of desensitization in an ENaC/degenerin channel, FASEB J 27(12) (2013) 5034-45.

[93] M.C. Della Vecchia, A.C. Rued, M.D. Carattino, Gating transitions in the palm domain of ASIC1a, J Biol Chem 288(8) (2013) 5487-95. 